\title{
NONCOMMUTATIVE ORLICZ MODULAR INEQUALITIES RELATED TO PARALLELOGRAM LAW
}

\section{ABDUGHENI ABDUREXIT}

Abstract. In this paper, we generalize the related parallelogram low inequalities to noncommutative Orlicz modular case. Besides, we give the $k$ sets of operators of related noncommutative parallelogram law inequalities.

Mathematics subject classification (2010): 46L51, 46A80.

Keywords and phrases: Von Neumann algebra, noncommutative Orlicz modular spaces, related parallelogram low inequalities.

\section{REFERENCES}

[1] W. B. ARveson, Analyticity in operator algebras, Amer. J. Math. 89 (1967), 578-642.

[2] G. PISIER, Q. XU, Noncommutative $L^{p}$-spaces, Handbook of the geometry of Banach spaces 2 (2003), 1459-1517.

[3] T. ANDo, X. ZHAN, Norm inequalities related to operator monotone functions, Math. Ann. 315 (1999), 771-780.

[4] T. FACK, H. Kosaki, Generalized s-numbers of $\tau$-measure operators, Pacific J. Math. 123 (1986), 269-300.

[5] M. S. Moslehian, M. Tominaga, K. Saito, Schatten p-norm inequalities related to an extended operator Parallelogram low, Linear Algebra Appl. 435 (2011), 823-829.

[6] O. Hirzallah, F. Kittaneh, M. S. Moslehian, Schatten p-norm inequalities related to a characterization of inner product spaces, Math. Inequal. Appl. 13 (2) (2010), 235-241.

[7] J. MusielaK, Orlicz spaces and modular spaces, in: Lecture Notes in Math. Vol. 1034, SpringerVerlag, 1983.

[8] A. AbDUREXIT, T. N. BEKJAN, Noncommutative Orlicz modular spaces associated with growth functions, Banach J. Math. Anal. 9 (4) (2015), 115-125.

[9] A. AbdureXit, T. N. BeKJan, Noncommutative Orlicz-Hardy spaces associated with growth functions, J. Math. Anal. Appl. 420 (1) (2014), 824-834.

[10] G. SADEGHI, Non-commutative Orlicz spaces associated to a modular on $\tau$-measurable operators, J. Math. Anal. Appl. 395 (2012), 705-715.

[11] M. S. Moslehian, G. S ADEghi, Inequalities for trace on $\tau$-measurable operators, Commun. Appl. Math. Comput. 28 (4) (2014), 379-389.

[12] R. Cheng, C. B. HARris, Duality of the weak parallelogram laws on Banach spaces, J. Math. Anal. Appl. 404 (1) (2013), 64-70.

[13] T. N. BeKJAn, D. DAUITBEK, Submajorization inequalities of $\tau$-measurable operators for concave and convex functions, Positivity 19 (2015), 341-345.

[14] M. S. Moslehian, An operator extension of the parallelogram law and related norm inequalities, Math. Inequal. Appl. 14 (2) (2011), 717-725. 